\title{
Caso clínico: Enfermedad Veno-Oclusiva Hepática secundaria a Quimioterapia en un paciente con Sarcoma de Ewing.
}

*Correspondencia:

raifgnn@gmail.com

Teléfono [593] 9988347429

Conflicto de intereses: Los autores declaran no tener conflictos de intereses.

Fondos: Ver la página 38

Recibido: 1 Septiembre 2017 Aceptado: 11 Noviembre 2017 Publicado: 30 Abril 2018

Membrete bibliográfico: Nasre-Nasser R, Correa M, Olmenares N, Sánchez M. Caso clínico: Enfermedad Venooclusiva Hepática secundaria a quimioterapia en un paciente con Sarcoma de Ewing. Rev. Oncol. Ecu 2018;28(1):34-40.

DOI: https://doi.org/10.33821/262

Copyright Nasre-Nasser, et al. Este artículo es distribuido bajo los términos de Creative Commons Attribution License, el cual permite el uso y redistribución citando la fuente y al autor original.

\section{Case report: Veno-Occlusive liver disease secondary to Chemotherapy in a patient with Ewing's sarcoma.}

\section{Raif Nasre Nasser ${ }^{\text {* }}$ iD, Magdalena Correa Vega², Norlis Olmenares Álvarez³, Mariener Sánchez Dugarte4.}

1. Instituto de Endocrinología, Metabolismo y Reproducción, Quito - Ecuador.

2. Hospital Universitario de Los Andes, Mérida - Venezuela, Servicio de Pediatría.

3. Hospital Materno Infantil "Samuel Darío Maldonado", Barinas - Venezuela, Servicio de Pediatría.

4. Hospital Regional de Talca, Talca - Chile, Servicio de Terapia intensiva Pediátrica.

\section{Resumen}

Introducción: La enfermedad veno-oclusiva hepática o síndrome de obstrucción sinusoidal se caracteriza clínicamente por presentar hepatomegalia dolorosa, ictericia, ascitis, edema y aumento de peso, que generalmente ocurre como una complicación del trasplante de médula ósea.

Caso clínico: Se trata de un escolar masculino de 9 años de edad con Sarcoma de Ewing quien presentó una Enfermedad Veno-oclusiva Hepática secundaria a la administración de quimioterapia con ciclofosfamida y vincristina.

Evolución: Se realizó el diagnóstico diferencial con daño hepático por causas infecciosas y autoinmunes a través de pruebas serológicas y biopsia.

Conclusión: Siendo este el primer reporte que describe la asociación entre la enfermedad Venooclusiva Hepática con Sarcoma de Ewing.

Palabras Claves: ENFERMEDAD VENO-OCLUSIVA HEPÁTICA, CICLOFOSFAMIDA, VINCRISTINA, SARCOMA DE EWING, PEDIATRÍA. 


\section{Abstract}

Introduction: Hepatic Veno-occlusive Disease or Sinusoidal Obstruction Syndrome is a clinical syndrome characterized by painful hepatomegaly, jaundice, ascites, edema and weight gain, which usually occurs as a complication of bone marrow transplantation.

Clinical Case: We describe a new case of male school age 9 with Ewing Sarcoma who had Hepatic Veno-occlusive Disease secondary to chemotherapy with cyclophosphamide and vincristine.

Evolution: We set the differential diagnosis from liver damage due to infection and autoimmune diseases through serological tests and biopsy.

Conclusion: This is the first report that describes the association between Ewing Sarcoma and Hepatic Veno-occlusive Disease.

Keywords: VENO-OCCLUSIVE HEPATIC DISEASE, CYCLOPHOSPHAMID, VINCRISTINE, EWING SARCOMA, PEDIATRICS.

DOI: $10.33821 / 262$

\section{Introducción}

La Enfermedad Veno-oclusiva Hepática (EVOH), también conocida como Síndrome de Obstrucción Sinusoidal (SOS) hepático, se caracteriza clínicamente por hepatomegalia dolorosa, ictericia y aumento de peso debido a retención hídrica [1]. Este cuadro clínico se produce por la hipertensión portal secundaria a la obliteración de los sinusoides hepáticos, que posteriormente puede comprometer las venas centrolobulillares del hígado [1, 2]. Por otro lado el sarcoma de Ewing es un tumor maligno de células redondas. Es una enfermedad rara en la cual las células neoplásicas se ubican en el hueso o en tejidos blandos. Las áreas afectadas con más frecuencia son la pelvis, el fémur, el húmero, y las costillas. El sarcoma de Ewing suele afectar a los niños y adultos jóvenes. Previamente no se había descrito la asociación de EVOH con el Sarcoma de Ewing por lo que es un diagnóstico muy infrecuente. A continuación se presenta un caso.

\section{Caso Clínico}

Se trata de un escolar masculino de 9 años de edad, quien presentó desde noviembre de 2006 un aumento del volumen del brazo izquierdo de manera progresiva, se acompaña de dolor de intensidad importante, motivo por el cual su madre consultó un mes después del inicio del cuadro. El paciente fue ingresado en diciembre de 2006 en el Hospital Universitario de Los Andes, con el diagnóstico de Sarcoma de Ewing estadio III. El tratamiento establecido fue quimioterapia con vincristina, ciclofosfamida, uromitexan, cardioxame y adriamicina, desde enero 2007 hasta agosto 2008. Luego de nueve meses de quimioterapia el paciente fue sometido a tumorectomía con colocación de injerto y síntesis 
de placa. Entre sus antecedentes el paciente presentaba alergia a la oxacilina, sin otros personales o familiares de importancia.

Posteriormente, en enero 2011 el paciente reingresó por presentar dolor abdominal, alzas térmicas, ictericia, acolia y episodios de emesis. Al examen físico de ingreso se evidenció: cicatriz posquirúrgica en el brazo izquierdo de $20 \mathrm{~cm}$ de longitud, tinte ictérico de piel y mucosas; abdomen distendido, blando con evidencia de ascitis, hepatomegalia a 3 centímetros del reborde costal derecho y dolor a la palpación del cuadrante superior derecho; resto sin alteraciones. Entre los hallazgos de los exámenes paraclínicos de ingreso se destacaron: hemoglobina $10.41 \mathrm{~g} / \mathrm{dl}$, hematocrito $29.7 \%$, leucocitos $3510 / \mathrm{mm}^{3}$, linfocitos $55.4 \%$, plaquetas $67 \mathrm{mil} / \mathrm{cm}^{3}$, bilirrubina total $6.87 \mathrm{mg} / \mathrm{dl}$, bilirrubina directa 4.47 $\mathrm{mg} / \mathrm{dl}$, tiempos de coagulación prolongados, aspartato aminotransferasa (AST) $363 \mathrm{U} / \mathrm{L}$ y alalina aminotransferasa (ALT) $246 \mathrm{U} / \mathrm{L}$.

Durante su estancia hospitalaria se le realizaron pruebas serológicas para detectar virus de hepatitis B y C, así como para el virus de inmunodeficiencia humana $(\mathrm{VIH})$, siendo estas negativas; asimismo, se descartaron causas de hepatitis autoinmune. Por otro lado, se le realizó ecografía abdominal con hallazgos compatibles de hepatomegalia, esteatosis pancreática, esplenomegalia y ascitis. En la endoscopía digestiva superior se evidenciaron varices esofágicas grado II, varices gástricas grado III y pangastropatía hipertensiva.

Tomando en consideración que aún no se conocía la causa del cuadro clínico, se decidió realizar una biopsia hepática percutánea en febrero 2011 con el fin de practicar estudio histopatológico. Éste reportó la pérdida total de la arquitectura del parénquima hepático, con presencia de micro y macronódulos separados por bandas de fibrosis en puente; nódulos de regeneración de hepatocitos desorganizados con ausencia de venas centrales y compuestos de células agrandadas focalmente dispuestas formando estructuras pseudoacinares, sin evidencia de malignidad. De este modo, se concluyó la presencia de una inflamación crónica leve e inespecífica en las zonas periportales con ausencia de infiltrado denso linfocítico que pudiera sugerir una etiología viral, autoinmune o eosinofílica; sugiriendo así una posible causa medicamentosa que al correlacionarse con el cuadro de hipertensión portal, ascitis, hepatomegalia e ictericia, así como el antecedente de haber recibido quimioterapia debido al Sarcoma de Ewing, orientó al diagnóstico de la $\mathrm{EVOH} / \mathrm{SOS}$.

\section{Evolución}

Con el diagnóstico de EVOH/SOS, el paciente recibió medidas de soporte que consistieron en dieta de protección hepática y administración de espironolactona de acuerdo al peso, con lo que mejoró la sintomatología y los valores de laboratorio del perfil hepático. Ante la estabilidad clínica del paciente se egresó de la institución, después de lo cual no se cuenta con datos sobre la evolución ambulatoria del paciente ya que el mismo no acudió a los controles médicos programados. 


\section{Discusión}

Actualmente, el mayor número de pacientes que desarrolla EVOH/SOS está estrechamente relacionado con la administración de quimioterapia durante el tratamiento del tumor de Wilms, del rabdomiosarcoma y de la leucemia linfoide aguda, en adición a aquellos casos en los que se presentan como complicación del trasplante de médula ósea [3, 4]. Por lo tanto, son poco frecuentes los reportes de esta entidad en pacientes pediátricos que reciben quimioterapia a dosis convencional para otros tipos de cáncer [4]; así como el presente caso, siendo a su vez el primer reporte de un paciente con Sarcoma de Ewing que desarrolla esta enfermedad.

La aparición de EVOH/SOS se vincula a múltiples factores de riesgo, entre los que destacan la edad (niños>adultos, en particular menores de 1 año), el sexo (mujeres>hombre), y la exposición a determinados fármacos hepatotóxicos como: ciclofosfamida, actinomicina $D$, oxaliplatino, azatioprina, 6-tioguanina, gemuzumab-ozogamicina, vincristina, gemcitabina, progestágenos, anfotericina B, ketokonazol, entre otros [5]. En el caso descrito, la ciclofosfamida y la vincristina utilizados en la quimioterapia por el Sarcoma de Ewing, han condicionado los cambios fisiopatológicos subyacentes al cuadro clínico; de igual modo, aunque esta enfermedad es más frecuente en mujeres y niños menores de un año, se ha presentado en un paciente masculino de 9 años de edad.

El estándar de oro para el diagnóstico de la $\mathrm{EVOH} / \mathrm{SOS}$ es el estudio histopatológico con obtención de tejido mediante biopsia hepática [6]; sin embargo, considerando la naturaleza invasiva y los potenciales riesgos del procedimiento se sostiene que el diagnóstico es principalmente clínico $[4,7]$ basado en los criterios de Seattle modificado [8], de Baltimore [9] o los recientemente acuñados por la Sociedad Europea de Sangre y Trasplantes de Médula [10].

El manejo de estos pacientes varía de acuerdo a la severidad del cuadro, siendo el tratamiento sintomático el indicado para aquellos casos leves o moderados considerando que un 80 a $90 \%$ presentan resolución espontánea. Mientras que para los pacientes con $\mathrm{EVOH} / \mathrm{SOS}$ severo además de las medidas de soporte ameritan tratamiento específico medicamentoso (por ejemplo: defibrotide) o quirúrgico (derivación porto-sistémica intrahepática transyugular o trasplante hepático) [6, 11-13].

\section{Conclusiones}

En resumen, se ha descrito el caso de un paciente con EVOH/SOS en un paciente con Sarcoma de Ewing, asociación no descrita hasta el momento. Por lo tanto, es de vital importancia identificar el riesgo de presentar este síndrome de acuerdo a la terapia que recibe el paciente y considerarlo entre las posibles causas de falla hepática cuando se haya administrado ciclofosfamida, vincristina o cualquier otro medicamento relacionado con la $\mathrm{EVOH} / \mathrm{SOS}$. 


\section{Agradecimientos}

Reconocemos a los familiares que autorizaron la publicación del presente caso clínico.

\section{Información adicional}

Nota del Editor

La Revista Oncología Ecu permanece neutral con respecto a los reclamos jurisdiccionales en mapas publicados y afiliaciones institucionales.

\section{Abreviaturas}

EVOH: Enfermedad Veno-oclusiva Hepática.

SOS: Síndrome de Obstrucción Sinusoidal.

\section{Archivos Adicionales}

Ninguno declarado por los autores.

\section{Fondos}

Los fondos de la investigación fueron propios de los autores del presente artículo. Cabe destacar que parte de la información inherente al caso clínico fue presentada en los siguientes eventos científicos:

- LVII Congreso Venezolano de Pediatría "Dra. Gloria Yamin de Barboza". Celebrado desde el 3 al 9 de septiembre de 2011 en la ciudad Maracaibo - Venezuela. (Ganador del primer lugar en el concurso de caso clínico).

- XXVI Congreso Científico Internacional FELSOCEM 2011. Celebrado desde el 6 al 10 de septiembre de 2011 en la ciudad Asunción - Paraguay

\section{Contribuciones de los autores}

RNN, MCV realizaron la idea de investigación, revisión bibliográfica. MCV, recolección de datos, escritura del artículo. NCA y MSD realizaron el análisis crítico del artículo. RNM realizó las correcciones editoriales. Todos los autores leyeron y aprobaron la versión final del artículo.

\section{Aprobación de ética y consentimiento para participar}

No aplica a este estudio. 
Se cuenta con el permiso escrito de sus padres para la publicación del presente estudio.

Información de los autores

Raif Nasre Nasser, médico cirujano, Instituto de Endocrinología, Metabolismo y iD Reproducción, Quito - Ecuador.* Correo electrónico: raifgnn@gmail.com https://orcid.org/0000-0002-8350-1008

Magdalena Correa Vega, médica pediatra subespecialista en infectología, Hospital Universitario de Los Andes, Mérida - Venezuela. Correo electrónico: magdalencorrea@gmail.com

Norlis Colmenares Álvarez, médica pediatra subespecialista en neonatología, Hospital Materno Infantil "Samuel Darío Maldonado", Barinas - Venezuela. Correo electrónico: norliscol@gmail.com

Mariener Sánchez Dugarte, médica pediatra subespecialista en terapia intensiva pediátrica, Hospital Regional de Talca, Talca - Chile.

\section{Referencias}

Abreviaturas en la referencias DOI: Digital Object Identifier

PMID: PubMed Identifier SU: Short URL
1. Carreras E. Prevención y tratamiento de la enfermedad venooclusiva hepática. Gastroenterol Hepatol 2011;34(9):635-40. SU: goo.gl/VpQAGG

2. DeLeve LD, Shulman HM, McDonald GB. Toxic Injury to Hepatic Sinusoids: Sinusoidal Obstruction Syndrome (Veno-Occlusive Disease). Semin Liver Dis. 2002;22(1):027-42. SU: goo.gl/AJmpMA

3. MacQuillan G, Mutimer D. Fulminant liver failure due to severe veno-occlusive disease after haematopoietic cell transplantation: a depressing experience. Q J Med 2004;97(9):581-9. SU: goo.gl/zK64hE

4. Kotecha R, Buckland A, Phillips MB, Cole CH, Gottardo NG. Hepatic SInusoidal Obstruction Syndrome During Chemotherapy for Childhood Medulloblastoma: Report of a Case and Review of the Literature. Clin Lab Obs 2014;36(1):76-80. PMID: 3872829

5. Valla DC, Cazals-Hatem D. Sinusoidal obstruction syndrome. Clin Res Hepatol Gastroenterol [Internet]. 2016;40(4):378-85. DOI: 10.1016/j.clinre.2016.01.006

6. Dalle J-H, Giralt SA. Hepatic Veno-Occlusive Disease after Hematopoietic Stem Cell Transplantation: Risk Factors and Stratification, Prophylaxis, and Treatment. Biol Blood Marrow Transplant [Internet]. 2016;22(3):400-9. SU: goo.gl/5gz97c

7. Kızılocak H, Dikme G, Özdemir N, Kuruğoğlu S, Adaletli I, Erkan T, et al. Sinusoidal Obstruction Syndrome During Chemotherapy of Pediatric Cancers and its Successful Management With Defibrotide. Clin Lab Obs 2017;39(7):373-6. PMID: 28859028

8. McDonald GB, Hinds MS, Fisher LD, Schoch HG, Wolford JL, Banaji M, et al. Veno-occlusive disease of the liver and multiorgan failure after bone marrow transplantation: a cohort study of 355 patients. Ann Intern Med 1993;118(4):255-67. PMID: 8420443 
9. Jones RJ, Lee KS, Beschorner WE, Vogel VG, Grochow LB, Braine HG, et al. Venoocclusive disease of the liver following bone marrow transplantation. Transplantation 1987;44(6):778-83. PMID: 3321587

10. Corbacioglu S, Carreras E, Ansari M, Balduzzi A, Cesaro S, Dalle J, et al. Diagnosis and severity criteria for sinusoidal obstruction syndrome/veno-occlusive disease in pediatric patients: a new classification from the European society for blood and marrow transplantation. Bone Marrow Transplant 2018;53(2):138-45. SU: goo.gl/WJjagN

11. Richardson PG, Riches ML, Kernan NA, Brochstein JA, Mineishi S, Termuhlen AM, et al. Phase 3 trial of defibrotide for the treatment of severe veno-occlusive disease and multi-organ failure. Blood 2016;127(13):1656-65. PMID: 26825712

12. Dignan FL, Wynn RF, Hadzic N, Karani J, Quaglia A, Pagliuca A, et al. BCSH/BSBMT guideline: diagnosis and management of veno-occlusive disease (sinusoidal obstruction syndrome) following haematopoietic stem cell transplantation. Br J Haematol 2013;163(4):444-57. DOI: 10.1111/bjh.12558

13. Koenecke C, Kleine M, Schrem H, Krug U, Nashan B, Neipp M, et al. Sinusoidal Obstruction Syndrome of the Liver after Hematopoietic Stem Cell Transplantation: Decision Making for Orthotopic Liver Transplantation. Int J Hematol 2006;83(3):271-4. PMID: 16720562 